\title{
Dermatomiosite como Primeiro Sinal de Neoplasia
}

\section{Dermatomyositis as the First Sign of a Neoplasia}

Márcia Mendonça Souto¹, Fernanda Linhares²

Autor Correspondente/Corresponding Author:

Márcia Mendonça Souto [m2souto@hotmail.com]

Rua Principal, Paredes, 5090-210 Palheiros, Murça, Portugal

ORCID iD: 0000000289084719

\section{RESUMO}

Os autores apresentam o caso de um homem de 69 anos, que dá entrada no serviço de urgência por diminuição da força muscular proximal com um mês de evolução. Apresentava ainda lesões cutâneas pruriginosas disseminadas no tronco, com o sinal $\mathrm{V}$, rash heliotrópico e erupção cutânea no dorso, face e membros superiores, tendo sido feito o diagnóstico de dermatomiosite. Do estudo analítico efetuado verificou-se anticorpos anti-nucleares positivos, elevação de creatina quinase, lactato desidrogenase e aldolase. A biópsia cutânea confirmou o diagnóstico. Do exame físico foi constatada uma massa no quadrante superior do abdómen e considerando poder tratar-se de síndrome paraneoplásica realizou ecografia, tomografia computorizada abdominal e ressonância magnética abdominal com identificação de massa única hepática com 12×8x7 cm. A biópsia desta lesão foi compatível com colangiocarcinoma. O doente faleceu de hemorragia intraparenquimatosa na região lenticocapsular externa, sem causa identificável. Com este caso pretende-se chamar a atenção da associação entre a dermatopolimiosite e tumores sólidos.

PALAVRAS-CHAVE: Colangiocarcinoma; Dermatomiosite; Síndromes Paraneoplásicas

1. Interna de $5^{\circ}$ ano de Medicina Interna, Centro Hospitalar de Trás-os-Montes e Alto Douro, Unidade de Chaves, Portugal. 2. Assistente Graduada Medicina Interna, Centro Hospitalar de Trás-os-Montes e Alto Douro, Unidade de Chaves, Portugal.

Recebido/Received: 30/05/2019 - Aceite/Accepted: 10/09/2019 - Publicado/Published: 30/09/2019

- Autor (es) (ou seu (s) empregador (es)) 2019. Reutilização permitida de acordo com CC BY-NC. Nenhuma reutilização comercial. ${ }^{\circ}$ Author(s) (or their employer(s)) 2019. Re-use permitted under CC BY-NC. No commercial re-use. 


\section{ABSTRACT}

The authors present a clinical case of a 69-year-old male that was admitted in the emergency department with decreased strength in proximal muscle that started in the previous month. He also presented erythematous and pruriginous cutaneous lesions disseminated along the torso, with V-shaped, heliotropic rash, and rash in dorsum, face and upper limbs. Dermatomyositis was diagnosed. He was antinuclear antibodies positive and the analytical study showed elevated creatine kinase, lactate dehydrogenase and aldolase. The skin biopsy also confirmed the diagnosis. In the abdominal examination a mass was noticed in the right upper quadrant. Considering the possibility of the presence of a paraneoplastic syndrome, the patient underwent an abdominal ultrasonography, computed tomography and magnetic resonance imaging that showed a single hepatic massive lesion $(12 \times 8 \times 7 \mathrm{~cm})$. The biopsy performed was compatible with cholangiocarcinoma. The patient died of intraparenchymal hemorrhage in the external lenticocapsular region, with no identifiable cause. With this clinical case it is intended to alert about the association between dermatomyositis and solid tumors.

KEYWORDS: Cholangiocarcinoma; Dermatomyositis; Paraneoplastic Syndromes

\section{INTRODUÇÃO}

A dermatomiosite (DM) é uma doença rara, progressiva com atingimento cutâneo e muscular. Os pacientes geralmente apresentam exantema generalizado e fraqueza muscular com limitação funcional, particularmente, nos músculos proximais. ${ }^{1}$ Os músculos do pescoço e faringe podem ser afetados, estando frequentemente associados a disfagia e dificuldade respiratória. A DM está frequentemente associada a neoplasias e, portanto, a sua identificação precoce pode preceder o diagnóstico correto de eventos neoplásicos. ${ }^{1-3}$

\section{CASO CLÍNICO}

Homem de 69 anos, caucasiano, casado. Antecedentes pessoais de hipertensão arterial essencial e diabetes mellitus tipo 2.

Recorre ao serviço de urgência por diminuição da força muscular, mais evidente nas cinturas escapulo-umeral e pélvica, com rigidez e edema articular, com cerca de um mês de evolução. Associadamente, apresenta erupção cutânea eritematosa, maculopapular na região malar, frontal, face ântero-superior do tronco (sinal em $\vee$ e si-

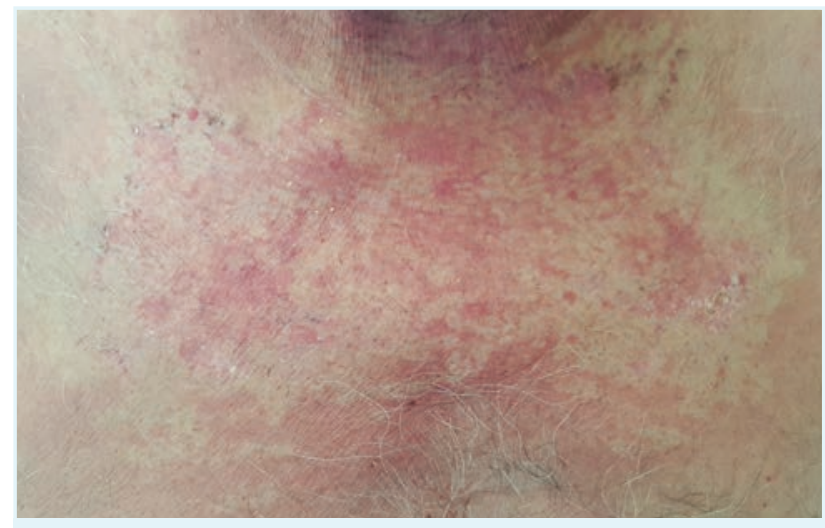

FIGURA 1. Lesões cutâneas de eritema - sinal em V. nal de xaile) e membros superiores (Fig. 1). Apresentava edema palpebral e eritema periorbitário (eritema heliotrópico) (Fig. 2). Ao exame físico constata-se, ainda, no quadrante superior direito do abdómen, uma massa com cerca de $10 \mathrm{~cm}$.

Analiticamente apresenta hemoglobina 12,7g/dL; leucócitos 7100/ul; AST 310U/L; ALT 115U/L; GGT 280U/L; FA 118U/L; CK 4363U/L; PCR 3,23 mg/dL; VS 60 mm/1ah; aldolase 21,0U/L; AgHBS positivo, com carga viral indetetável.

Dado o quadro clínico, o doente, fica internado para complementar o estudo.

Durante o internamento, e pensando no diagnóstico de DM, efetuou-se estudo para despiste e eventual associação a doença autoimune/neoplasia. $\bigcirc$ estudo imunológico evidenciou anticorpos anti-nucleares (ANA) 1:160 (positivos); anti-SSA 37,0U/mL; FR 15Ul/mL; anti-M2 1,3U/mL. A biópsia cutânea/muscular revelou infiltrado linfohistocitário perivascular com hiperplasia do endotélio, compatível com DM. A ecografia abdominal e posteriormente a tomografia computorizada (TC) e ressonância magnética (RM) confirmaram a presença

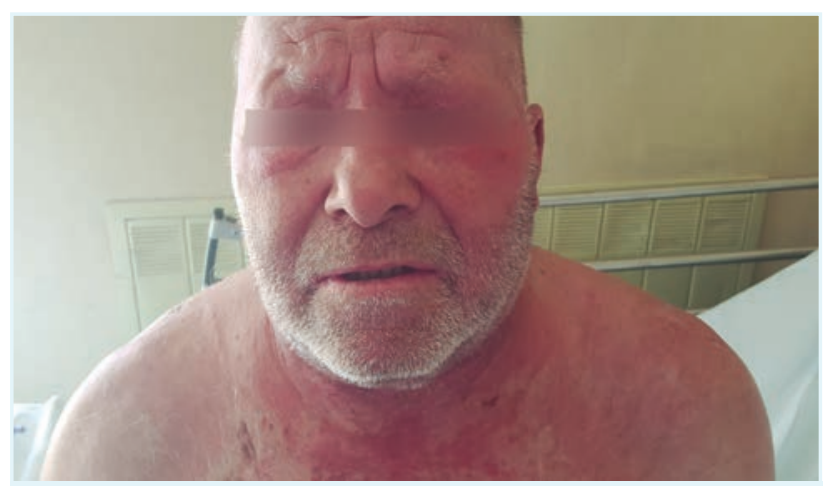

FIGURA 2. Edema palpebral e eritema periorbitário (eritema heliotrópico). 
de uma massa hepática com $12 * 8 * 7 \mathrm{~cm}$ (Fig. 3). Estudo endoscópico com endoscopia digestiva alta e baixa sem alterações. A biópsia hepática foi compatível com adenocarcinoma e o estudo imunohistoquímico com colangiocarcinoma (Fig. 4). Não realizou eletromiografia dada as limitações físicas apresentadas pelo doente.

Neste período de internamento, houve agravamento significativo da dermatomiosite com disfunção faríngea, disfagia intensa, principalmente para sólidos, disfonia, com compromisso da via aérea superior por abaulamento do palato mole. Efetuou corticoterapia sistémica com prednisolona $1 \mathrm{mg} / \mathrm{kg} /$ dia e posteriormente, por agravamento da sintomatologia realizou imunoglobulinas 0,4 g/kg/dia e bólus de 1000 mg metilprednisolona.

Feito o diagnóstico e DM em contexto paraneoplásico por colangiocarcinoma com metastização hepática, foi transferido para o Serviço de Oncologia do Hospital, para início de quimioterapia paliativa, que não chegou a ser efetuada, dado o doente ter apresentado disartria e hemiparesia esquerda, com evidência imagiológica por TC-cerebral de hematoma núcleo capsular direito com colapso do ventrículo lateral homolateral e desvio da linha média, sem indicação cirúrgica. $\bigcirc$ doente veio a falecer no decorrer deste internamento.

\section{DISCUSSÃO}

A DM é uma doença do tecido conjuntivo que se associa a miopatia, diminuição da força muscular proximal e manifestações cutâneas, nomeadamente, pápulas de Gottron, rash heliotrópico, edema periorbitário, eritema reticular, poiquilodermia e erupção pápulo-escamosa. O diagnóstico definitivo é dado pela presença de alterações cutâneas características, associadas a debilidade muscular e confirmação histológica. ${ }^{4}$ Os critérios de Bohan e Peter incluem: 1. Fraqueza muscular proximal e simétrica. 2. Elevação sérica das enzimas musculares (creatina quinase, aldolase, lactato desidrogenase, transaminase glutâmico-oxaloacética e transaminase

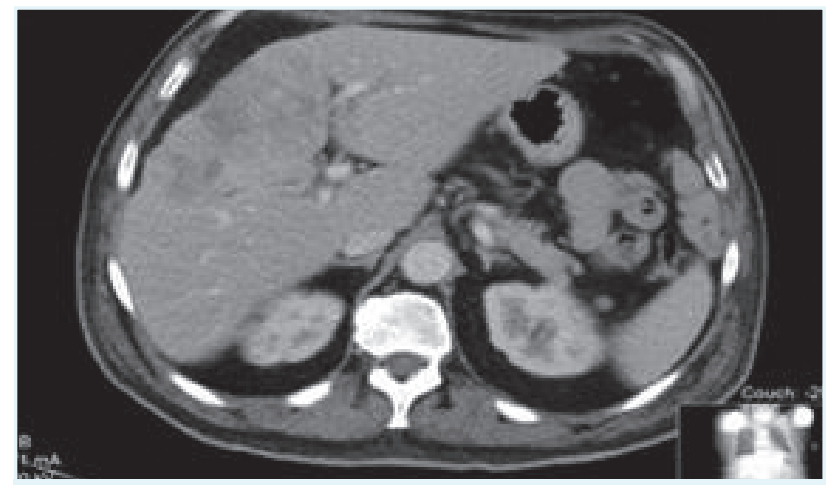

FIGURA 3. Imagem de tomografia computorizada de lesão hepática multinodular com $12 \mathrm{~cm}$ de maior diâmetro. glutâmico-pirúvica). 3. Alterações miopáticas na eletromiografia. 4. Biopsia muscular com alterações características de miosite. Podem estar ainda presentes sintomas sistémicos, artralgias, sinovite, disfagia e alterações gastrointestinais com má absorção e dismotilidade, alterações pulmonares, por diminuição da força muscular respiratória e doença intersticial, alterações cardíacas e vasculares, nomeadamente vasculite e fenómenos de Raynaud.1,2 Em 2017, os critérios de classificação foram atualizados pelas European League Against Rheumatism/American College of Rheumatology (EULAR/ACR), sendo definido o diagnóstico de miopatias idiopáticas inflamatórias, como definitivo, provável ou possível, sendo que para o diagnóstico de DM, se estiverem presentes lesões cutâneas patognomónicas, tais como, pápulas de Gottron, rash heliotrópico e/ou sinal de Gottron, a biopsia cutânea deixa de ser essencial para o diagnóstico. ${ }^{5}$

A etiologia da dermatomiosite permanece desconhecida, mas parece resultar de processos mediados por alterações imunológicas iniciadas por fatores como malignidade, drogas e agentes infeciosos em indivíduos geneticamente predispostos. ${ }^{6}$

A evidência de reação inflamatória e vasculite, a presença de autoanticorpos e miotoxicidade mediada por célula T ou microangiopatia mediada pelo complemento, a associação com outras doenças autoimunes, sugerem uma natureza autoimune da dermatomiosite. 1,6,7

A DM está associada a um risco, até três vezes superior de neoplasia, principalmente em pacientes com idade superior a 45 anos, podendo a neoplasia ser diagnosticada antes, durante ou após o diagnóstico da dermatomiosite. ${ }^{2,8}$

Quando associada a neoplasia pode manifestar-se com uma maior extensão de lesões cutâneas, prurido persistente, manifestações atípicas da doença cutânea (presença de ulceração com bolhas ou vasculite) e maior resistência ao tratamento convencional, sendo que se verifica uma melhoria após o tratamento da neoplasia

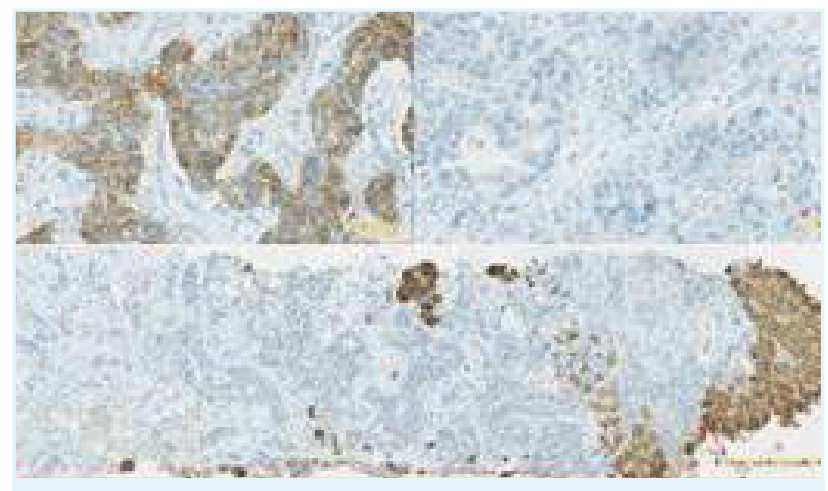

FIGURA 4. Imunohistoquímica de biópsia hepática compatível com colangiocarcinoma. 
e reaparecimento da fraqueza muscular quando há recidiva do tumor., 90 anticorpo anti-155/140 tem demonstrado uma forte predileção para a coexistência de malignidade, principalmente quando associado ao HLA-DQ1A*0301. ${ }^{7}$

As neoplasias mais frequentemente diagnosticadas em associação com a DM são: ovário, pulmão, pâncreas, estômago, colorretal e linfoma não-Hodgkin.

As seguintes características, quando presentes ao diagnóstico, estão associadas a pior prognóstico: sexo masculino, idade avançada, fraqueza muscular proximal, disfagia, hipoventilação, doença intersticial pulmonar, baixo nível sérico de proteína-quinase, associação com neoplasia, presença de auto-anticorpos anti-SRP e anti-155/140. Já as causas mais comuns de morte prematura são a infeção, neoplasia, doença cardiovascular e doença intersticial pulmonar.?

No caso descrito, estamos perante um doente que apresentava ao diagnóstico vários fatores indicadores de mau prognóstico, nomeadamente, idade avançada, sexo masculino, fraqueza muscular proximal, disfagia e associação com doença neoplásica, já com metastização hepática no momento do diagnóstico. Apesar de o doente vir a falecer de causa, aparentemente, não relacionada com a neoplasia, foi notório o acentuar do mau estado geral e debilidade física, durante o internamento. Assim, deve-se estar sempre atento aos sinais clínicos sugestivos de DM, permitindo o diagnóstico mais célere de uma possível doença neoplásica associada.

CONFLITOS DE INTERESSE: Os autores declaram não ter qualquer conflito de interesse na realização do presente trabalho.

FONTES DE FINANCIAMENTO: Não houve qualquer fonte de financiamento na realização do presente trabalho.

CONFIDENCIALIDADE DOS DADOS: Os autores declaram ter seguido os protocolos da sua instituição acerca da publicação dos dados de doentes.

CONSENTIMENTO: Consentimento do doente para publicação obtido.

PROVENIÊNCIA E REVISÃO POR PARES: Não comissionado; revisão externa por pares.

CONFLICTS OF INTEREST: The authors declare that they have no conflicts of interest.

FINANCIAL SUPPORT: This work has not received any contribution, grant or scholarship.

CONFIDENTIALITY OF DATA: The authors declare that they have followed the protocols of their work center on the publication of data from patients.

PATIENT CONSENT: Consent for publication was obtained.

PROVENANCE AND PEER REVIEW: Not commissioned; externally peer reviewed.

\section{REFERÊNCIAS}

1. Dalakas MC, Hohlfeld R. Polymyositis and dermatomyositis. Lancet. 2003;362:971-82. doi: 10.1016/S01406736(03)14368-1.

2. Hill CL, Zhang Y, Sigurgeirsson B, Pukkala E, Mellemkjaer L, Airio $A$, et al. Frequency of specific cancer types in dermatomyositis and polymyositis: a population-based study. Lancet. 2001;357:96-100. doi: 10.1016/S0140-6736(00)03540-6.

3. Chen YJ, Wu CY, Huang YL, Wang CB, Shen JL, Chang YT. Cancer risks of dermatomyositis and polymyositis: a nationwide cohort study in Taiwan. Arthritis Res Ther. 2010; 12:R70. doi: 10.1186/ar2987.

4. Cervera R, Espinosa G, Ramos R, Hernândez-Rodrigues J, Did M. Enfermidades autoinmunes sistémicas Diagnóstico y tratamento. $5^{a}$ ed. Madrid: Editorial médica Panamericana; 2014.

5. Lundberg IE, Tjärnlund A, Bottai M, Werth VP, Pilkington C, de Visser M, et al. 2017 European League Against Rheumatism/ American College of Rheumatology classification criteria for adult and juvenile idiopathic inflammatory myopathies and their major subgroups. Ann Rheum Dis. 2017;69:2271-82. doi: 10.1002/art.40320.

6. Shamim EA, Rider LG, Miller FW. Update on the genetics of the idiopathic inflammatory myopathies. Curr Opin Rheumatol. 2000;12:482-91.

7. Brandão M, Marinho A. Idiopathic inflammatory myopathies: Definition and management of refractory disease. Autoimmun Rev. 2011;10:720-4. doi: 10.1016/j.autrev.2011.05.021.

8. Madan V, Chinoy H, Griffiths CE, Cooper RG. Defining cancer risk in dermatomyositis. Part I. Clin Exp Dermatol. 2009;34:451-5. doi: 10.1111/j.1365-2230.2009.03216.x.

9. Tournadre A, Dubost J, Soubrier M. Treatment of inflammatory muscle disease in adults. Joint Bone Spine .2010; 77: 3904. doi: 10.1016/j.jbspin.2010.04.007.

10. Stockton D, Doherty V. Risk of cancer in patients with dermatomyositis or polymyositis, and follow-up implications: a Scottish population-based cohort study. Br J Cancer. 2001; 85:415. doi: 10.1054/bjoc.2001.1699. 